\title{
Nuclear Imaging of Cancer Cell Therapies
}

\author{
Vladimir Ponomarev
}

Department of Radiology, Memorial Sloan-Kettering Cancer Center

A promising role of cellular therapies in cancer treatment is reflected by the constantly growing number of clinical trials with adoptively transferred cells. Direct and indirect cell labeling for the nuclear imaging of transferred cells has been proven reliable for imaging adoptive cellular therapies. Both methods show their advantages and limitations. Direct labeling is a relatively easy, inexpensive, and well-established methodology. Indirect labeling using a reporter gene imaging paradigm allows for reliable, stable, and harmless visualization of cellular trafficking, persistence, proliferation, and function at the target site. It is expected that new human-derived reporter genes will be rapidly translated into clinical applications that require repetitive imaging for the effective monitoring of various genetic and cellular therapies.

Key Words: molecular imaging; oncology; PET/CT; cell therapy; reporter gene

J Nucl Med 2009; 50:1013-1016

DOI: 10.2967/jnumed.109.064055

Recent developments in biotechnology have led to an increasing number of clinically available anticancer therapies that require the administration of cells of different origins. These cellular therapies are based on the ex vivo manipulation of various therapeutically useful cell types (e.g., stem cells, lineage progenitors, mesenchymal cells, lymphocytes, and dendritic cells), followed by local or systemic administration to cancer patients (1-4). A promising role of cellular therapies in cancer treatment, especially in minimal residual disease and micrometastases, is reflected by the constantly growing number of clinical trials with adoptively transferred cells (5). Until recently, immunostaining of whole-body slices of small animals was the most straightforward, reliable, and traditional approach used for assessing the localization and targeting of adoptively transferred cells (6). New methods for the noninvasive and repetitive evaluation of trafficking, homing, tumor targeting, differentiation, and persistence of adoptively transferred cells would significantly aid the development of progenitor or effector cell-based therapy approaches and facilitate their clinical implementation. For example, systemic administration of antigen-specific T lymphocytes is one of the most studied and clinically applicable

\footnotetext{
Received Oct. 6, 2008; revision accepted Mar. 10, 2009.

For correspondence or reprints contact: Vladimir Ponomarev, Department of Radiology, Memorial Sloan-Kettering Cancer Center, 1275 York Ave., Z-2063 New York, NY 10021.

E-mail: ponomarv@mskcc.org

COPYRIGHT @ 2009 by the Society of Nuclear Medicine, Inc.
}

methods of adoptive anticancer cell therapy. Long-term trafficking and localization of T lymphocytes is an important component of anticancer immune response and of elimination of abnormal cells and infectious agents from the body. Imaging could help monitor such processes as T cell migration, homing to the tumor target, and long-term $\mathrm{T}$ cell viability and their subsequent activation and cytolytic activity. These questions could potentially be answered by repetitive imaging using clinically applicable techniques, for example, nuclear imaging.

\section{EX VIVO RADIOLABELING FOR NUCLEAR IMAGING}

The first experiments on noninvasive imaging of lymphocyte trafficking were performed with extracorporal labeling. These methods were applied to different immune cells using various radioisotopes for labeling. ${ }^{111} \mathrm{In}$, in particular, found a wide clinical application in oncology as an imaging agent for monitoring immunotherapy with tumor-infiltrating lymphocytes. In a study published by Fisher et al. (7), 6 patients with metastatic malignant melanoma who had multiple sites of subcutaneous, nodal, or visceral disease were treated with ${ }^{111}$ Inlabeled tumor-infiltrating lymphocytes (TILs) (mean dose, $8.4 \times$ $10^{9}$ labeled cells per patient), followed by serial whole-body $\gamma$-camera imaging and serial biopsies of tumor and normal tissue. ${ }^{111}$ In-labeled TILs localized to lung, liver, and spleen within $2 \mathrm{~h}$ after the infusion. This study showed that labeled TILs can localize preferentially to tumors, and it provided information about the possible mechanism of the therapeutic effects of TILs. In another study, autologous monocyte-derived dendritic cells loaded with a tumor antigen and labeled with ${ }^{111}$ In-oxine were injected intradermally in patients with varying cancer types $(8)$. Only 1 of the 6 patients treated had nodular inguinal uptake on the intradermally injected side, suggesting that dendritic cells rapidly moved to a regional lymph node in which antigen presentation should occur. Imaging techniques that involve ex vivo labeling of lymphocytes or other adoptively transferred cells have several limitations (e.g., the relatively low attainable level of radioactivity per cell when cells are labeled with passively equilibrating radiotracers such as ${ }^{111}$ In-oxine). In the study described above by Fisher et al. (7), the mean concentration of radioactivity of $144.3 \mathrm{kBq} / 10^{8}$ cells $\left(3.9 \mu \mathrm{Ci} / 10^{8}\right.$ cells $)$ was achieved with a total of $10,471 \mathrm{kBq}(283 \mu \mathrm{Ci})$ of ${ }^{111} \mathrm{In}$ activity injected. Significantly higher levels of radioactivity per cell could be obtained with tracers such as ${ }^{18} \mathrm{~F}-\mathrm{FDG}$ that use facilitated transport and enzyme-amplified accumulation. However, ${ }^{18} \mathrm{~F}$-FDG has been shown to gradually efflux out of the labeled cells ( 9 ) because of progressive loss of the radiolabel that 
occurs during cell division in vivo. Although more stable labeling of lymphocytes was achieved with ${ }^{125}$ I-5-iodo-2' -deoxyuridine, which is stably incorporated into the DNA of labeled cells (10), such an approach has never been tested. Another shortcoming of the ex vivo radiolabeling approach is the limited period of monitoring, which is due to radiolabel decay, cell division, and biologic clearance. In addition, the exposure of cells to higher doses of radioactivity during labeling may also be limited by radiotoxicity. In a study by Zanzonico et al. (11), T lymphocytes that were specific for the human anti-Epstein-Barr virus (EBV) were genetically engineered to express herpes simplex virus type 1 thymidine kinase (HSV1-tk) and radiolabeled ex vivo with ${ }^{131}$ I-2-fluoro-2-deoxy-5-iodo-1-D-arabinofuranosyluracil ( $\left.{ }^{131} \mathrm{I}-\mathrm{FIAU}\right)$ for $2 \mathrm{~h}$ at concentrations between 1 and 1,850 $\mathrm{kBq} / \mathrm{mL}$ (between 1 and $50 \mu \mathrm{Ci} / \mathrm{mL}$ ). At median nuclear absorbed doses up to $830 \mathrm{cGy}$, a ${ }^{51} \mathrm{Cr}$-release assay against B-lymphoblastoid cells showed no loss of immune cytotoxicity, thus demonstrating the functional integrity of tumor-reactive T cells labeled at this dose level for in vivo cell trafficking and tumor-targeting studies in the animals.

\section{Genetic Labeling of Cells with Reporter Genes for} Nuclear Imaging Using in Vivo Tracer Administration

Stable genetic labeling of adoptively transferred cells with various reporter genes has been used to circumvent the temporal limitations of ex vivo radiolabeling. The HSV1-tk reporter gene has been used in most seminal studies on imaging cell trafficking by nuclear techniques including imaging in humans (12-15). This paradigm involves the administration of a radiolabeled nucleoside analog probe that is selectively phosphorylated and trapped by interaction with the HSV1-tk enzyme in the transduced cell. In contrast to the above-mentioned (11) study, in which HSV1-tk-expressing T cells were prelabeled with ${ }^{131} \mathrm{I}$ FIAU ex vivo, Koehne et al. (16) demonstrated the feasibility of long-term (at least for several weeks) PET monitoring of targeting and migration of EBV-specific cytotoxic $\mathrm{T}$ cells (CTLs) that are transduced to constitutively express a radionuclide-based HSV1-tk reporter gene for in vivo ${ }^{124}$ I-FIAU labeling. PET images acquired at $28 \mathrm{~h}, 8 \mathrm{~d}$, and $15 \mathrm{~d}$ after T cell infusion demonstrated high accumulations of $\mathrm{T}$ cells in the human leukocyte antigen-matched EBV-positive autologous and allogeneic tumors that resulted in the elimination of tumor xenografts. This paradigm provides the opportunity for repeated visualization of transferred $\mathrm{T}$ cells within the same animal over time using PET for continuous monitoring of $\mathrm{T}$ cell localization and persistence. Therapeutic antitumor immunity depends on a highly migratory CTL population capable of activation and trafficking between lymphoid and tumor-bearing microanatomic sites. Studies conducted by the UCLA group showed a specific targeting of the murine sarcoma virus antigenpositive tumor by mouse antigen-specific $\mathrm{T}$ cells that were transduced with the HSV1-sr39tk PET reporter gene (17). Specific localization of immune $T$ cells to the antigen-positive tumor was detected over time by sequential imaging with 9-[4- ${ }^{18}$ F-fluoro-3-(hydroxymethyl)butyl]guanine ( $\left.{ }^{18} \mathrm{~F}-\mathrm{FHBG}\right)$ of the same animals for $10 \mathrm{~d}$. Interestingly, in this study tumor growth was assessed by ${ }^{18} \mathrm{~F}$-FDG scans. By using two ${ }^{18} \mathrm{~F}$-based radiotracers with rapid clearance and a radioisotope with a short half-life, repeated PET of tumor progression and $\mathrm{T}$ cell trafficking can be performed on a daily basis, thus providing accurate quantitative assessment of $\mathrm{T}$ cell dynamics and tumor response in vivo. PET can be used to kinetically measure the induction and therapeutic modulations of cell-mediated immune responses. Shu et al. showed the feasibility of PET to monitor the effect of immunosuppressive drugs on immune cell populations in the draining lymph nodes using hematopoietic stem and progenitor cells transduced with the HSV1-sr39tk, synthetic Renilla luciferase, and enhanced green fluorescent protein (GFP) trifusion reporter gene (18). It was shown that activation and expansion of immune cell populations in the draining lymph nodes could no longer be detected after mice were treated with dexamethasone. New genetic strategies aim to enhance cellular antitumor immunity in cancer patients by genetic reprogramming used to improve $\mathrm{T}$ cell survival, augment cell expansion, and offset $\mathrm{T}$ cell anergy and immune suppression. A recent study by Dobrenkov et al. (19) was the first to combine both genetic therapeutic (prostate cancer-specific chimeric antigen receptor [CAR]) and PET (HSV1-tk reporter gene and ${ }^{18} \mathrm{~F}-\left[2^{\prime}\right.$-deoxy2 '-fluoro- $\beta$-D-arabinofuranosyl]-5-ethyluracil) components using genetic modifications of primary human $\mathrm{T}$ lymphocytes. This therapeutic or imaging strategy provides spatial and temporal information on CAR-positive $\mathrm{T}$ cell trafficking and persistence and can be used for antigen-specific CAR-transduced $\mathrm{T}$ cell treatment monitoring and prediction of tumor response and therapy outcome. Bone marrow transplantation (BMT) is one of the most radical and effective approaches in cancer treatment, not only in hematologic malignancies but also in solid tumors. This approach allows for the successful displacement of affected bone marrow (BM) of the recipient with donor hematopoietic cells. Nuclear imaging can identify the initial skeletal engraftment sites after BMT. Using a standard mouse model of BMT, Mayer-Kuckuk et al. (20) introduced a combined optical and PET reporter gene, triple-fusion encoding for HSV1-tk, GFP, and Firefly luciferase into mouse BM cells. PET was applied to acquire 3-dimensional images of the wholebody in vivo biodistribution of the transplanted cells, followed by CT. Coregistration of PET and CT mapped the sites of BM engraftment, and multiple, discrete BM cell engraftment sites were observed. With the noninvasive monitoring of BM engraftment using this multimodality approach, BMT can be tailored to the individual needs of patients, with minimal toxicity of conditioning regimens, sufficient numbers of transplanted cells, and adequate cytokine support. A similar approach has been used for the monitoring of mesenchymal stem cell targeting of microscopic tumors and tumor stroma development (21). As imaged by PET and ${ }^{18} \mathrm{~F}-\mathrm{FHBG}$, tracer human mesenchymal stem cells expressing HSV1-tk and GFP reporter genes could target microscopic tumors, subsequently proliferate and differentiate, and contribute to the formation of a significant portion of tumor stroma. Therefore, PET should facilitate clinical translation of stem cell-based anticancer gene therapeutic approaches by providing the means for in vivo noninvasive whole-body monitoring of trafficking, tumor targeting, and proliferation of reporter gene-expressing tracer human mesen- 
chymal stem cells in tumor stroma. The major impediment to the translation of virus- and bacterium-derived reporter gene imaging approaches into clinical practice is the immunogenicity of these non-human-derived reporter proteins. This is especially important when the repetitive administration of a reporter gene or long-term monitoring of transgene expression is required, which could potentially be compromised by an immune reaction against this foreign protein. To circumvent this problem, several human-derived intrinsically nonimmunogenic reporter genes have been proposed for nuclear imaging, including the dopamine receptor type 2 (22), sodium iodide symporter (23), human mitochondrial thymidine kinase type 2 (24), and human norepinephrine transporter (hNET) (25). In the latter study, Doubrovin et al. (25) evaluated hNET, permitting the imaging of transduced cells in vivo with an approved clinical-grade radiolabeled probe (metaiodobenzylguanidine), and demonstrated the feasibility of nuclear imaging of 2 different cell populations using 2 independent reporter genes (Fig. 1).

\section{FUNCTIONAL IMAGING OF CELLS}

Recently, several groups have demonstrated that the noninvasive reporter gene imaging of endogenous molecular genetic processes is feasible with PET. An essential component of the

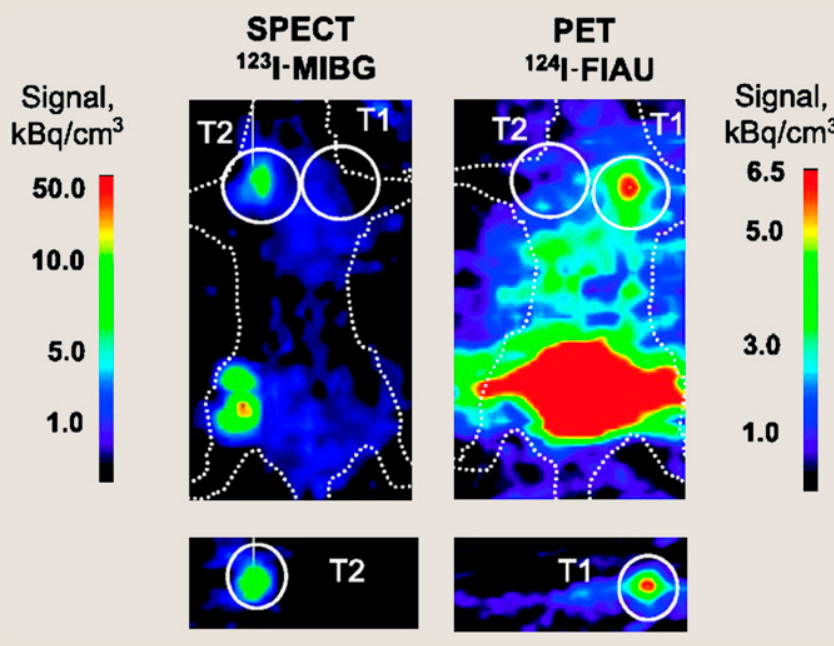

FIGURE 1. Dual-modality imaging using hNET and HSV1-tk reporter genes for monitoring different populations of antigenspecific CTLs. Severe combined immunodeficient mice bearing 2 subcutaneous xenografts-HLA-A0201+EBVBLCL (T1) and HLA-DRB10701+EBV-BLCL (T2)-received intravenous infusion of mixture of EBV-specific CD4+CTLhNET $\left(15 \times 10^{6}\right.$ cells per mouse), restricted by HLADRB10701 and CD8+CTL-HSV1-tk $\left(9 \times 10^{6}\right.$ cells per mouse), restricted by HLA-A0201 allele. To avoid ${ }^{124}$ I downscatter into 123| SPECT energy window, mice were first injected with ${ }^{123}$ MIBG and imaged $4 \mathrm{~h}$ thereafter with SPECT. This image showed selective accumulation of 123 -MIBG in T2 tumor targeted by CD4+CTL-hNET. No signal above background was detected in T1 tumor. After completion of SPECT, ${ }^{124}$ IFIAU was given. Mice were then imaged by PET $2 \mathrm{~h}$ later. In these PET images, only T1 tumor was visualized. No signal was detected in T2 tumor. HLA = human leukocyte antigen; hNET = human norepinephrine transporter; MIBG = metaiodobenzylguanidine. (Reprinted with permission of (25).) anticancer immune response is tumor-specific $\mathrm{T}$ cell activation. Ponomarev et al. (26) have monitored and assessed T cell activation in vivo using an activation-sensitive HSV1-tkencoding genetic reporter system and PET with ${ }^{124}$ I-FIAU. The results of the study demonstrated that PET of $\mathrm{T}$ lymphocyte activation after $T$ cell receptor engagement is feasible using the described reporter system. Furthermore, this activation could be arrested pharmacologically by the administration of clinically used calcineurin inhibitors such as cyclosporin A and FK506. This circumstance makes this imaging approach clinically valuable, because it can be used in patients receiving immunosuppressive drugs after BM and organ transplantation. Ultimately, the process of immune cell activation can be visualized using a direct radiotracer approach. In a recent study, Radu et al. (27) demonstrated that $1-\left(2^{\prime}\right.$-deoxy-2'-18 $\mathrm{F}$-fluoroarabinofuranosyl) cytosine $\left({ }^{18} \mathrm{~F}-\mathrm{FAC}\right)$ was specific for imaging activation-specific upregulation of the deoxyribonucleotide salvage pathway in lymphoid cells (Fig. 2). ${ }^{18} \mathrm{~F}$-FAC enabled visualization of lymphoid organs and was sensitive to localized immune activation in a mouse model of antitumor immunity. PET detected early changes in lymphoid mass in systemic autoimmunity and allowed the evaluation of immunosuppressive therapy. Such an approach can be readily translated into clinical practice for the noninvasive PET monitoring of immune cell activation during the antitumor immunologic response.

\section{QUANTITATION OF ADOPTIVELY TRANSFERRED CELLS IN VIVO}

The degree of PET signal in an anatomic region of interest (ROI) largely depends on the density of labeled cells in a volume of tissue. The potential of PET for quantifying cell signals in ROIs provides a unique opportunity to estimate the absolute number of injected labeled cells at the target site. However, little is known about the constraints and parameters for using PET signal detection to establish cell numbers in different ROIs. Su et al. (28) determined the correlation of PET signal to cell number and characterized the cellular limit of detection for PET. This study, using native mouse T cells transduced with the HSV1-tk reporter gene and PET with ${ }^{18} \mathrm{~F}-\mathrm{FHBG}$, revealed a cell numberdependent signal, with a limit of detection calculated as $10^{6}$ cells in an ROI (0.1-mL volume). In another study (29), memory cells demonstrated early accumulation and apparent proliferation with large $\mathrm{T}$ cell numbers at the antigen-positive tumor as early as day 1 after $T$ cell transfer. Both naive and memory cells eradicated the antigen-expressing tumor at a comparable density of intratumoral $\mathrm{T}$ cells $\left(2-4 \times 10^{6} / \mathrm{g}\right)$. These methods and findings provide a strategy for quantitation of cellularity using PET that has implications for both experimental models and clinical diagnosis.

\section{CONCLUSION AND FUTURE CONSIDERATIONS}

Direct and indirect cell labeling for the nuclear imaging of transferred cells has been proven reliable for imaging adoptive cellular therapies. Both methods show their advantages and limitations. Direct labeling is a relatively easy, inexpensive, and well-established methodology. High-contrast cell-to-medium or -tissue ratios can be achieved easily. However, this approach is limited by a short period of monitoring (days), which is due to 
FIGURE 2. PET/CT of immune cell activation using ${ }^{18} \mathrm{~F}-\mathrm{FAC}$ radiotracer. Increased ${ }^{18} \mathrm{~F}$-FAC retention in spleen and lymph nodes at peak of primary antitumor immune response. ${ }^{18} \mathrm{~F}-\mathrm{FAC}$ PET/CT of mice on day $-1(A)$ and day 15 (B) after oncoretrovirus injection. At peak of immune response (day 15), increased ${ }^{18} \mathrm{~F}-\mathrm{FAC}$ retention is seen in lymph nodes and spleen. (C) Quantification of ${ }^{18} \mathrm{~F}-\mathrm{FAC}$ retention in spleen and lymph nodes on day -1 and day $15(n=3)$. (D) ${ }^{18} \mathrm{~F}-\mathrm{FDG}$ PET/CT of mice on day 13 after challenge. ${ }^{18} \mathrm{~F}$ FDG retention is seen in lymph nodes,

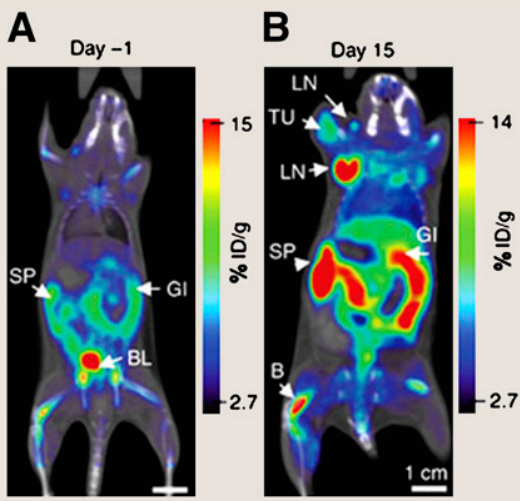

C

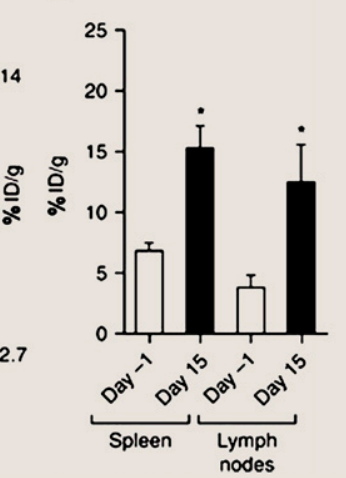

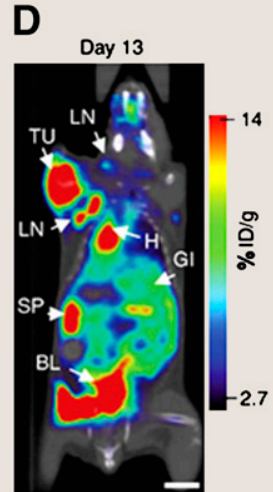

tumor, and spleen. \%ID/g = percentage injected dose per gram; $\mathrm{B}=$ bone; $\mathrm{BL}=$ bladder; $\mathrm{Gl}=$ gastrointestine; $\mathrm{H}=$ heart; $\mathrm{LN}=$ lymph node; SP = spleen; TU = tumor. (Reprinted with permission of (27).)

radiolabel decay, cell division, and biologic clearance. High levels of radiolabel concentration can impair the biologic functioning of the labeled cells. Indirect labeling using a reporter gene imaging paradigm allows for the reliable, stable, and harmless visualization of cellular trafficking, persistence, proliferation, and function at the target site. Genetically labeled cells can be repetitively imaged for a long period of observation (months). Picomolar concentrations of radiotracer administered in vivo are harmless for the labeled cells and for the surrounding organs and tissues. The genetic nature of this method (stable integration into the cell genome) permits in vivo labeling of the progeny of injected cells. The functional imaging of cell activation, cytokine signaling, and proapoptotic events is feasible and can be performed in preclinical experiments and in clinical settings using inducible genetic reporter systems and the direct tracer approach. It is expected that new human-derived reporter genes will be rapidly translated into clinical applications that require repetitive imaging for the effective monitoring of various genetic and cellular therapies.

\section{REFERENCES}

1. Erlandsson A, Morshead CM. Exploiting the properties of adult stem cells for the treatment of disease. Curr Opin Mol Ther. 2006;8:331-337.

2. Keating A. Mesenchymal stromal cells. Curr Opin Hematol. 2006;13:419-425.

3. Rooney C, Smith CA, Ng CY, et al. Use of gene-modified virus-specific T lymphocytes to control Epstein-Barr-virus-related lymphoproliferation. Lancet. 1995;345:9-13.

4. Buchsel PC, DeMeyer ES. Dendritic cells: emerging roles in tumor immunotherapy. Clin J Oncol Nurs. 2006;10:629-640.

5. Prados J, Melguizo C, Boulaiz H, Marchal JA, Aranega A. Cancer gene therapy: strategies and clinical trials. Cell Mol Biol. 2005;51:23-36.

6. Reinhardt R, Jenkins MK. Whole-body analysis of T cell responses. Curr Opin Immunol. 2003;15:366-371.

7. Fisher B, Packard BS, Read EJ, et al. Tumor localization of adoptively transferred indium-111 labeled tumor infiltrating lymphocytes in patients with metastatic melanoma. J Clin Oncol. 1989;7:250-261.

8. Blocklet D, Toungouz M, Kiss R, et al. ${ }^{111} \mathrm{In}$-oxine and ${ }^{99 \mathrm{~m}} \mathrm{Tc}-\mathrm{HMPAO}$ labelling of antigen-loaded dendritic cells: in vivo imaging and influence on motility and actin content. Eur J Nucl Med Mol Imaging. 2003;30:440-447.

9. Adonai $\mathrm{N}$, Nguyen $\mathrm{KN}$, Walsh $\mathrm{J}$, et al. Ex vivo cell labeling with ${ }^{64} \mathrm{Cu}-$ pyruvaldehyde-bis(N4-methylthiosemicarbazone) for imaging cell trafficking in mice with positron-emission tomography. Proc Natl Acad Sci USA. 2002;99:3030-3035.

10. Schelper RL, Adrian EK Jr. Monocytes become macrophages; they do not become microglia: a light and electron microscopic autoradiographic study using 125-iododeoxyuridine. J Neuropathol Exp Neurol. 1986;45:1-19.
11. Zanzonico P, Koehne G, Gallardo HF, et al. [ $\left.{ }^{131} \mathrm{I}\right] \mathrm{FIAU}$ labeling of genetically transduced, tumor-reactive lymphocytes: cell-level dosimetry and dose-dependent toxicity. Eur J Nucl Med Mol Imaging. 2006;33:988-997.

12. Gambhir SS, Herschman HR, Cherry SR, et al. Imaging transgene expression with radionuclide imaging technologies. Neoplasia. 2000;2:118-138.

13. Jacobs A, Voges J, Reszka R, et al. Positron-emission tomography of vectormediated gene expression in gene therapy for gliomas. Lancet. 2001;358:727-729.

14. Penuelas I, Mazzolini G, Boan JF, et al. Positron emission tomography imaging of adenoviral-mediated transgene expression in liver cancer patients. Gastroenterology. 2005;128:1787-1795.

15. Yaghoubi SS, Jensen MC, Satyamurthy N, et al. Noninvasive detection of therapeutic cytolytic T cells with ${ }^{18} \mathrm{~F}$-FHBG PET in a patient with glioma. Nat Clin Pract Oncol. 2009;6:53-58.

16. Koehne G, Doubrovin M, Doubrovina E, et al. Serial in vivo imaging of the targeted migration of human HSV-TK-transduced antigen-specific lymphocytes. Nat Biotechnol. 2003;21:405-413.

17. Dubey $\mathrm{P}, \mathrm{Su} \mathrm{H}$, Adonai N, et al. Quantitative imaging of the $\mathrm{T}$ cell antitumor response by positron-emission tomography. Proc Natl Acad Sci USA. 2003;100:1232-1237.

18. Shu C, Guo S, Kim YJ, et al. Visualization of a primary anti-tumor immune response by positron emission tomography. Proc Natl Acad Sci USA. 2005;102:17412-17417.

19. Dobrenkov K, Olszewska M, Likar Y, et al. Monitoring the efficacy of adoptively transferred prostate cancer-targeted human $\mathrm{T}$ lymphocytes with PET and bioluminescence imaging. J Nucl Med. 2008;49:1162-1170.

20. Mayer-Kuckuk P, Doubrovin M, Bidaut L, et al. Molecular imaging reveals skeletal engraftment sites of transplanted bone marrow cells. Cell Transplant. 2006;15:75-82.

21. Hung S, Deng WP, Yang WK, et al. Mesenchymal stem cell targeting of microscopic tumors and tumor stroma development monitored by noninvasive in vivo positron emission tomography imaging. Clin Cancer Res. 2005;11:7749-7756.

22. MacLaren D, Gambhir SS, Satyamurthy N, et al. Repetitive, non-invasive imaging of the dopamine D2 receptor as a reporter gene in living animals. Gene Ther. 1999;6:785-791.

23. Groot-Wassink T, Aboagye EO, Glaser M, Lemoine NR, Vassaux G. Adenovirus biodistribution and noninvasive imaging of gene expression in vivo by positron emission tomography using human sodium/iodide symporter as reporter gene. Hum Gene Ther. 2002;13:1723-1735.

24. Ponomarev V, Doubrovin M, Serganova I, et al. A novel non-immunogenic reporter gene for non-invasive imaging in humans: human thymidine kinase type 2 [abstract]. J Nucl Med. 2002;43(5 suppl):70P.

25. Doubrovin MM, Doubrovina ES, Zanzonico $P$, et al. In vivo imaging and quantitation of adoptively transferred human antigen-specific $\mathrm{T}$ cells transduced to express a human norepinephrine transporter gene. Cancer Res. 2007;67:11959-11969.

26. Ponomarev V, Doubrovin M, Lyddane C, et al. Imaging TCR-dependent NFATmediated T-cell activation with positron emission tomography in vivo. Neoplasia. 2001;3:480-488.

27. Radu CG, Shu CJ, Nair-Gill E, et al. Molecular imaging of lymphoid organs and immune activation by positron emission tomography with a new $\left[{ }^{18} \mathrm{~F}\right]$-labeled 2'-deoxycytidine analog. Nat Med. 2008;14:783-788.

28. Su H, Forbes A, Gambhir SS, Braun J. Quantitation of cell number by a positron emission tomography reporter gene strategy. Mol Imaging Biol. 2004;6:139-148.

29. Su H, Chang DS, Gambhir SS, Braun J. Monitoring the antitumor response of naive and memory CD8 T cells in RAG1-/- mice by positron-emission tomography. J Immunol. 2006;176:4459-4467. 